\title{
ON APPROXIMATION OF FUNCTION IN GENERALIZED ZYGMUND CLASS USING $C^{\eta} T$ OPERATOR
}

\section{H. K. NIGAM AND MD. HADISH}

\begin{abstract}
In the present work, we, for the first time, study the error estimates of a function $h\left(2 \pi\right.$-periodic) in generalized Zygmund class $Z_{r}^{u}(r \geqslant 1)$ by Cesàro-Matrix $\left(C^{\eta} T\right)$ product means of its Fourier series (F. S.). The results obtained in the paper provide the best approximation of the function $h$ in $Z_{q}^{u}(q \geqslant 1)$ class. Our Theorem 2.1 generalizes eight previously known results. Thus, the results of Singh and Srivastava [29], Lal and Kushwaha [25], Lal [23], Nigam and Sharma [10], Nigam [8], Lal [22] and Ug̃ur Deg̃er [28] become the particular cases of our Theorem 2.1. Several useful results in the form of corollaries are also achieved from the main theorems.
\end{abstract}

Mathematics subject classification (2010): 41A10,41A25,42B05,42A50,40G05,40C05.

Keywords and phrases: Error estimates, generalized Zygmund class, Cesàro-Matrix operator, Fourier series.

\section{REFERENCES}

[1] A. Zygmund, Trigonometric series, Cambridge Univ. Press, Cambridge, 3rd rev. ed., 2002.

[2] B. E. RHAODES, On the degree of approximation of functions belonging to Lipschitz class by Hausdorff means of its Fourier series, Tamkang Journal of Mathematics, 34 (3), 2003, 245-247.

[3] B.N. Sahney AND D.S. Goel, On the degree of continuous functions, Ranchi University Math. Jour. 4, 1973, 50-53.

[4] C. K. ChUI, An Introduction to Wavelets. Wavelet Analysis and Its Applications, vol. 1, Academic Press, San Diego 1992.

[5] G. ALEXITS, Convergence problems of orthogonal series, Translated from German by I Folder. International series of Monograms in Pure and Applied Mathematics Vol. 20, 1961.

[6] G. H. HARdy, Divergent Series, Oxford University Press, 1949.

[7] H. H. Khan, On degree of approximation of functions belonging to the class Lip $(\alpha, p)$, Indian J. Pure Appl. Math. 5 (3), 1974, 132-136.

[8] H. K. Nigam, Degree of approximation of a function belonging to weighted $\left(L_{r}, \xi(t)\right)$ class by $(C, 1)(E, q)$ means, Tamkang J. of Mathematics, vol. 42 (1), 2011, 31-37.

[9] H. K. NIGAM, Degree of approximation of functions belonging to Lip $\alpha$ class and weighted $\left(L_{r}, \xi(t)\right)$ class by product summability method, Surveys Math. Appl. 5, 2010, 113-122.

[10] H. K. Nigam AND K. Sharma, Degree of approximation of a class of a function by $(C, 1)(E, q)$ means of Fourier series, IAENG International Journal of Applied Mathematics, 41:2, 2011.

[11] J. K. KUShWAHA AND B. P. DhaKAL, Approximation of a function belonging to Lip $(\alpha, r)$ class by $N_{p, q} \cdot C_{1}$ summability method of its Fourier series, Nepal Journal of Science and Technology Vol. 14 (2), 2013, 117-122.

[12] K. J. Ans ARI, M. Mursaleen And S. Rahman, Approximation by Jakimovski-Leviatan operators of Durrmeyer type involving multiple Appel polynomials, Revista de la Real Academia de Ciencias Exactas, Físicas y Naturales. Series A. Matemáticas, vol-113(2), 1007-1024.

[13] K. S. TIWARI AND C. S. BARIWAL, The degree of approximation of functions in the Hölder metric by Triangular matrix method of Fourier series, international J. of pure and applied Mathematics Volume 76 (2)2012, 227-232. 
[14] K. QURESHI, On the degree of approximation of a function belonging to the class Lip $\alpha$, Indian J. pure Appl. Math. 13 (8), 1982, 898-903.

[15] K. QURESHI, On the degree of approximation of a periodic function $f$ by almost N'orlund means, Tamkang J. of Mathematics 12 (1), 1981, 35-38.

[16] K. QUREShi AND H. K. NEHA, A class of functions and their degree of approximation, Ganita. 41 (1), 1990, 37-42.

[17] L. LeINDLER, Trigonometric approximation of functions in $L_{p}$ norm, J. Math. Anal. Appl. $302,2005$.

[18] M. Mursaleen, K. J. Ansari And M. Nasiruzzaman, Approximation by q-analogue of Jackimovski-Leviatan operators involving q-Appell polynomials, Iranian J. of Science and Technology, Transactions A: Science, vol-41(4), Dec 2017, 891-900.

[19] M. Murs aleen, S. Rahman And K. J. Ans ARI, Approximation by generalized Stancu type integral operators involving Sheffer polynomials, Carpathian J. of Mathematics, 34(2), 2018, 215-228.

[20] O. TÖEPLITZ, Uberallagemeine lineara Mittelbil dunger P.M.F.22, 1913, 113-119.

[21] P. Chandra, Trigonometric approximation of functions in L p norm, J. Math. Anal. Appl. 275 (1), 2002, 13-26.

[22] S. LAL, On degree of approximation of functions belonging to the weighted $\left(L^{P}, \xi(t)\right)$ class by $(C, 1)(E, 1)$ means, Tamkang Journal of Mathematics Vol 30, 1999, 47-52.

[23] S. LAL, Approximation of functions belonging to the generalized Lipschitz class by $C^{1} . N_{p}$ summability method of Fourier series, Appl. Math. Comput. 209, 2009, 346-350.

[24] S. Lal And A. Mishra, The method of summation $(E, 1)\left(N, p_{n}\right)$ and Trigonometric Approximation of function in generalized Holder metric, J. of Indian Math. Soc. Vol 80 (1-2), 2013, 87-98.

[25] S. Lal AND J. K. KushwaHa, Degree of approximation of Lipschitz function by product summability method, International Mathematical Forum, 4 (43), 2009, 2101-2107.

[26] S. N. BERNSHTEIN, On the best approximation of continuous functions by polynomials of given degree, in: Collected Works [in Russian], Izd. Nauk SSSR, Moscow Vol. 1, 1952, pp. 11-104.

[27] U. Singh, M. L. MitTAL AND S. SOnKAR, Trigonometric approximation of signals (function) belonging to $W\left(L^{r}, \xi(t)\right)$-class by matrix $C^{1} . N_{p}$ operator, Int. J. Math Sci 2012 (2012) 1-11.

[28] U. DEG̃ER, On approximation to the functions in the $W\left(L_{p}, \xi(t)\right)$ class by a new matrix mean, Novi Sad J. Math. Vol. 46, (1), 2016, 1-14.

[29] U. Singh AND S. K. SRIVASTAVA, Trigonometric approximation of functions belonging to certain Lipschitz, classes by $C^{1} T$ operator, Asian-European J. Math., Vol. 7(4), 2014, 1450064. 